\title{
ESTUDO DAS DISPERSÕES AQUOSAS DE NANOTUBOS DE CARBONO UTILIZANDO DIFERENTES SURFACTANTES
}

Isabella R. da Silva e Pedro L. M. Barreto*

Departamento de Ciências e Tecnologia de Alimentos, Universidade Federal de Santa Catarina, 88040-900 Florianópolis - SC, Brasil Ismael C. Bellettini

Departamento de Química, Universidade Federal de Santa Catarina, 88040-900 Florianópolis - SC, Brasil

Recebido em 7/12/11; aceito em 9/8/12; publicado na web em 27/11/12

\begin{abstract}
STUDY OF AQUEOUS DISPERSIONS OF CARBON NANOTUBES USING DIFFERENT SURFACTANTS. The dispersion of carbon nanotubes in water for their utilization in nanoscale devices is a challenging task. Comparative studies on interaction and dispersion of multi-wall carbon nanotubes (MWNT) using two different surfactants (sodium dodecyl sulfate, SDS, and polyoxyethylenesorbitanmonooleate, Tween 80) are presented. The interaction between carbon nanotubes and surfactants was studied by tensiometry, conductivimetry, and fluorimetry. The dispersions of MWNT in surfactants were characterized using a UV-vis spectrophotometer. For effective dispersion, the minimum weight ratio of MWNT to surfactant was 1:41 and 1:3 for SDS and Tween 80, respectively.
\end{abstract}

Keywords: carbon nanotube; surfactant; dispersion.

\section{INTRODUÇÃO}

Os nanotubos de carbono (CNTs) foram observados pela primeira vez por Iijima, ${ }^{1}$ em 1991, e desde então têm recebido muita atenção devido às suas propriedades físicas exclusivas. ${ }^{2,3}$ Os CNTs exibem excelentes propriedades mecânica, elétrica, magnética e térmica, diâmetro de escala nanométrica, bem como razão de aspecto alta ( 1000) em comparação com os silicatos lamelares ( 200), que os tornam um elemento ideal de reforço para compósitos de polímeros. ${ }^{3,4}$

Em diversas das suas importantes aplicações, os CNTs somente apresentam suas propriedades únicas se estiverem em uma dispersão altamente homogênea. ${ }^{5}$ No entanto, CNTs são pouco solúveis na maioria dos solventes. Devido à forte atração de van der Waals, eles exibem uma tendência a se agregarem e formarem grandes agregados de difícil dispersão. ${ }^{6}$

No intuito de obter dispersões homogêneas, duas alternativas diferentes estão atualmente sendo utilizadas para dispersar os nanotubos de carbono, por exemplo, métodos mecânicos (físicos) ou métodos químicos. O uso de métodos mecânicos, como ultrassonicação e agitação com alta taxa de cisalhamento, permite a separação dos nanotubos uns dos outros. Além destes métodos fragmentarem os nanotubos durante o processo, causando a diminuição da razão de aspecto, também são métodos ineficientes e consomem tempo e energia elevados. Os métodos químicos usam surfactantes ou modificações químicas para alterar a energia de superfície dos CNTs, melhorando as características de molhabilidade e adesividade, e aumentam a estabilidade da dispersão no solvente. A funcionalização covalente inclui a ligação de diferentes grupos funcionais nas paredes dos nanotubos de carbono. No entanto, funcionalizações químicas agressivas causam um aumento nos defeitos nas paredes laterais, o que pode alterar as propriedades mecânicas e elétricas dos CNTs..$^{7-10}$

Uma ampla variedade de surfactantes (aniônico, catiônico e não iônico) tem sido estudada para dispersões de CNTs, como lauril sulfato de sódio (SDS), ${ }^{11,12}$ dodecil benzeno sulfato de sódio (SDBS), ${ }^{13}$ brometo de cetiltrimetil amônio $(\mathrm{CTAB}),{ }^{14}$ brometo de dodeciltrimetil amônio (DTAB) ${ }^{15}$ e a família dos surfactantes Tween. ${ }^{8}$ Os estudos

*e-mail: barreto@cca.ufsc.br indicam que os surfactantes produzem um eficiente recobrimento da parede do CNT e induzem repulsões eletroestáticas e/ou estéricas, que poderiam contrabalancear as atrações de van der Waals entre os CNTs. ${ }^{16,17}$ De acordo com Vaisman et al. ${ }^{18}$ e Bystrzejewski et al. ${ }^{10}$ os surfactantes adsorvem sua cauda hidrofílica na superfície do CNT através de forças de atração não covalentes. Os surfactantes em solução podem se organizar e formar agregados supramoleculares (micelas). A formação de micelas ocorre apenas acima da concentração micelar crítica $(\mathrm{cmc})$. No entanto, segundo Madni et al., ${ }^{9}$ geralmente é necessária uma alta concentração de surfactantes para se obter dispersões estáveis de CNTs, que é inconveniente devido ao alto custo dos surfactantes, seus impactos ambientais e por restringir o uso destas suspensões em materiais compósitos. ${ }^{9}$

Considerando a relevância dos surfactantes nas dispersões de CNTs, o objetivo do presente estudo foi comparar dois surfactantes, SDS e Tween 80, para dispersão de nanotubos através das técnicas de tensão superficial, condutividade, fluorescência e espectroscopia de UV-vis. O poder de dispersão dos surfactantes foi analisado experimentalmente, bem como teoricamente, com base em sua organização estrutural. Este estudo fornece insights sobre alguns parâmetros para a otimização da dispersão de nanotubos de multicamada (MWNTs) usando surfactantes. UV-vis foi empregado para analisar a capacidade de dispersão desses surfactantes e o uso de diferentes técnicas permitiu estabelecer uma relação MWNTs-surfactante para se obter uma dispersão ideal.

Do nosso estudo, a relação CNT-surfactante ideal acaba por ser o parâmetro mais importante na dispersão de nanotubos.

\section{PARTE EXPERIMENTAL}

\section{Materiais e métodos}

\section{Materiais}

Os nanotubos de carbono (CNTs) utilizados foram obtidos da Sigma-Aldrich com pureza $>95 \%$, diâmetro de 6-9 nm e comprimento de $5 \mu \mathrm{m}$. O dodecil sulfato de sódio (SDS), o mono-oleato de sorbitan (Tween 80) e o pireno têm procedência da Sigma-Aldrich, com pureza de $99 \%$. Ambos foram utilizados sem purificação prévia. 


\section{Tensão superficial}

As medidas de tensão superficial foram feitas utilizando um tensiômetro da Kruss, GMBH, modelo K6, com escala de divisão de $0,1 \mathrm{mN} \mathrm{m}^{-1}$ equipado com um anel de $\mathrm{Pt}-\mathrm{Ir}-20$. Foram usados volumes de 10,0 mL de solução do correspondente surfactante ou CNT-surfactante, previamente preparada para cada medida.

\section{Fluorescência}

As medidas de fluorescência do pireno foram executadas em água destilada. Uma solução de pireno de $1,0 \times 10^{-6} \mathrm{~mol} \mathrm{~L}^{-1}$ (utilizase essa concentração de pireno para evitar a formação de dímeros (excímeros)) foi preparada a partir de uma solução estoque de $1,0 \mathrm{x}$ $10^{-3} \mathrm{~mol} \mathrm{~L}{ }^{-1}$ em etanol. As soluções de Tween 80 foram preparadas a partir da solução contendo pireno, assim como as soluções de CNT. As soluções estoques de CNT-Tween 80 foram preparadas com homogenizador Ultra-Turrax ${ }^{\circledR}$ ((IKA) T25 digital) e sonicadas por $3 \mathrm{~h}$, utilizando-se um sonicador MaxiClean, modelo 1650A, frequência $20 \mathrm{~Hz}$.

Os espectros de fluorescência do estado estacionário do pireno foram determinados em um espectrofluorímetro Hitachi F-4500 equipado com uma cela de quartzo de $1,0 \mathrm{~cm}$, sob agitação magnética. As fendas de excitação e emissão do monocromador foram ajustadas para 10,0 e 5,0 nm, respectivamente. As amostras foram excitadas em 336,0 $\mathrm{nm}$ e os espectros de emissão foram corridos de 360,0 a 500,0 nm. Tipicamente, os espectros de fluorescência foram realizados após a adição de volumes conhecidos da solução estoque de surfactante. Para cada leitura, a concentração era corrigida devido à diluição ocorrida na cela que previamente continha $1,0 \mathrm{~mL}$ de solução contendo igual concentração de pireno. A razão $I_{1} / I_{3}$ foi estimada analisando-se a relação da intensidade máxima do pico em $372,8 \mathrm{~nm}\left(\mathrm{I}_{1}\right)$ e $384,0 \mathrm{~nm}\left(\mathrm{I}_{3}\right)$.

\section{Condutividade}

As medidas de condutividade específica foram realizadas utilizando-se um condutivímetro modelo Hanna, modelo HI 4321. As soluções contendo surfactante e/ou CNT-surfactante foram previamente preparadas para cada medida.

\section{Espectroscopia $U V$-vis}

As dispersões foram analisadas por um espectrômetro de ultravioleta visível da Hitachi, modelo U-1800 em um comprimento fixo de $260 \mathrm{~nm} ;{ }^{12}$ as medidas foram feitas incrementando-se a concentração do surfactante e mantendo-se a concentração de CNT. O branco foi realizado para cada nova amostra, com a concentração de surfactante puro correspondente.

\section{RESULTADOS E DISCUSSÃO}

Foram estudados dois sistemas para a dispersão dos CNTs em solução aquosa utilizando dois surfactantes, um aniônico (SDS) e outro não iônico (Tween 80). As técnicas utilizadas para estudar a interação entre os surfactantes e o CNT foram tensão superficial, condutividade, fluorescência e espectroscopia UV-vis.

\section{Sistema CNT + SDS}

\section{Tensão superficial}

Através de medidas de tensão superficial podem-se obter os valores de $c m c$ de surfactantes. ${ }^{19}$ Acima da $c m c$, as micelas, ao contrário dos monômeros, ficam dispersas em toda solução, sem efeito adicional sobre a tensão superficial da solução. Sabe-se que concentrações acima da $\mathrm{cmc}$ não apresentam um aumento significativo de moléculas de surfactante na interface ar-líquido e não têm qualquer influência sobre a tensão superficial, que permanece constante, somente contribuindo para a formação de mais micelas em solução. ${ }^{19,20}$

A Figura 1 apresenta a tensão superficial versus concentração de SDS na presença de $0,20 \mathrm{mg} \mathrm{mL}^{-1}$ de CNT. O gráfico de tensão superficial de SDS sem o CNT (Figura 1S, material suplementar) apresentou um mínimo, possivelmente devido à presença de dodecanol, ${ }^{21} \mathrm{e} o$ valor encontrado de $c m c$ aparente para o SDS foi de 6,2 $\mathrm{mmol} \mathrm{L}^{-1}$, diferente do encontrado na literatura pela mesma técnica para o SDS puro. ${ }^{22,23}$ De acordo com Lin et al., ${ }^{24}$ a existência de um mínimo ou um pico negativo no gráfico g versus $\log \mathrm{C}$ pode implicar que o surfactante é uma mistura, mas nenhum pico negativo no gráfico g versus $\log \mathrm{C}$ não implica que não há nenhuma impureza no surfactante ou que o surfactante seja constituído por um único componente. Uma ruptura clara com nenhum pico negativo no gráfico $\gamma$ versus $\log \mathrm{C}$ pode ser um dos melhores critérios de pureza surfactante, mas ainda não é uma garantia.

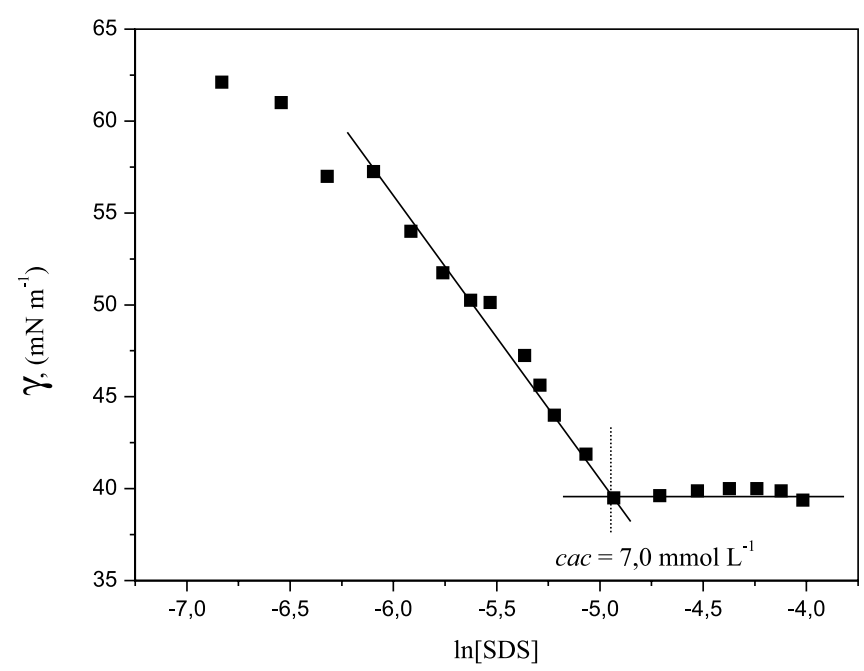

Figura 1. Tensão superficial versus concentração de SDS na presença de 0,20 $m g ~ m L^{-1}$ de CNT em solução aquosa

Para um sistema contendo nanotubos de carbono e SDS em solução aquosa, existe um equilíbrio dinâmico entre as moléculas de surfactante livres em solução, as localizadas na interface ar-líquido e aquelas adsorvidas na superfície dos CNTs. ${ }^{20}$ Quando a tensão superficial foi realizada na presença de $0,20 \mathrm{mg} \mathrm{mL}^{-1}$ de CNT (Figura 1), não apresentou um mínimo, sugerindo que o dodecanol pode ter sido adsorvido na parede do nanotubo por interação hidrofóbica. O valor encontrado para a concentração de agregação crítica ( $c a c$ ) foi de $7,0 \mathrm{mmol} \mathrm{L}^{-1}$, este aumento (comparado à cmc aparente) se deve ao fato de algumas moléculas de surfactante adsorverem hidrofobicamente à parede dos nanotubos de carbono ${ }^{25}$ que estão em solução, levando à diminuição da tensão interfacial CNT-água. ${ }^{26}$ As moléculas de SDS podem orientar-se perpendicularmente às paredes do nanotubo e formar filmes, ${ }^{27}$ sendo necessária uma maior concentração de surfactante para a formação das micelas, ${ }^{14,25}$ como representado na Figura 2.

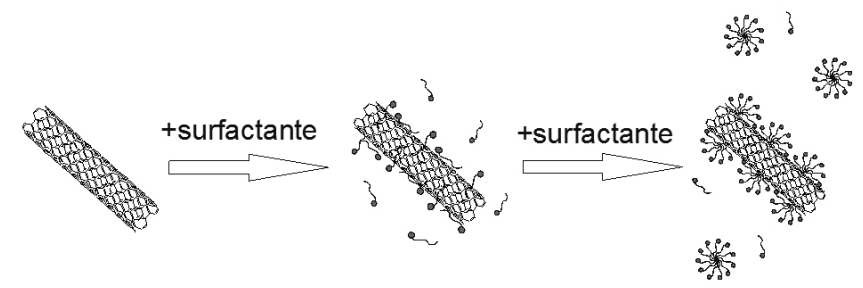

Figura 2. Representação esquemática da associação de surfactante com nanotubo de carbono 
A determinação das tensões superficiais em função da concentração de surfactante permite determinar além da $\mathrm{cmc}$ a concentração em excesso na superfície $\left(\Gamma_{\max }\right)$, pela equação de adsorção de Gibbs (Equação 1), ${ }^{28}$ e a área mínima por molécula de surfactante, $\mathrm{A}_{\min }$, na interface ar/solvente pela Equação $2 .{ }^{19}$

$$
\begin{gathered}
\gamma=-\Gamma_{\max } R T \ln C \\
A_{\min }=\frac{1}{\Gamma_{\max } N_{A}}
\end{gathered}
$$

onde, $\mathrm{R}$ é a constante dos gases, $\mathrm{N}_{\mathrm{A}}$ é o Número de Avogadro, $\gamma$ é o valor da tensão superficial e $\mathrm{C}$ é a concentração de surfactante na solução. Através destas equações podem-se determinar os parâmetros de adsorção interfacial do SDS puro $\left(\Gamma_{\max }=5,45 \times 10^{-6} \mathrm{~mol} \mathrm{~m}^{-2}\right.$, a $\mathrm{A}_{\text {min }}=30,5 \AA^{2}$ molécula $\left.^{-1}\right)$, assim como para o CNTS-SDS $\left(\Gamma_{\max }=\right.$ $5,95 \times 10^{-6} \mathrm{~mol} \mathrm{~m}^{-2}$, a A $_{\min }=27,9 \AA^{2}$ molécula $\left.^{-1}\right)$. A presença de CNT faz com que a área ocupada por molécula tenha uma diminuição comparada ao SDS puro, isto é uma evidência de que há um melhor empacotamento de moléculas de surfactante na superfície quando o nanotubo de carbono está presente na solução.

\section{Condutividade}

Medidas de condutividade elétrica também são utilizadas para acompanhar o processo de associação micelar e interação surfactantepolímero. ${ }^{29-31}$ A Figura 3 mostra o perfil da condutividade específica (em escala relativa) versus concentração de SDS na ausência e na presença de $0,20 \mathrm{mg} \mathrm{mL}^{-1}$ de CNT em solução aquosa. Observa-se que com o aumento na concentração de SDS, a condutividade específica também aumenta linearmente até o ponto de descontinuidade na curva, que define a $c m c$ em $7,8 \mathrm{mmol} \mathrm{L}^{-1}$. Com adição de mais SDS, a condutividade específica continua aumentando mais em uma taxa de variação menor do que aquela apresentada antes da $\mathrm{cmc}$. Na presença de $0,20 \mathrm{mg} \mathrm{mL}^{-1}$ de CNT o perfil da curva é muito parecido, mas com o ponto de descontinuidade em uma concentração maior do que para o SDS puro, $c a c=8,3 \mathrm{mmol} \mathrm{L}^{-1}$.

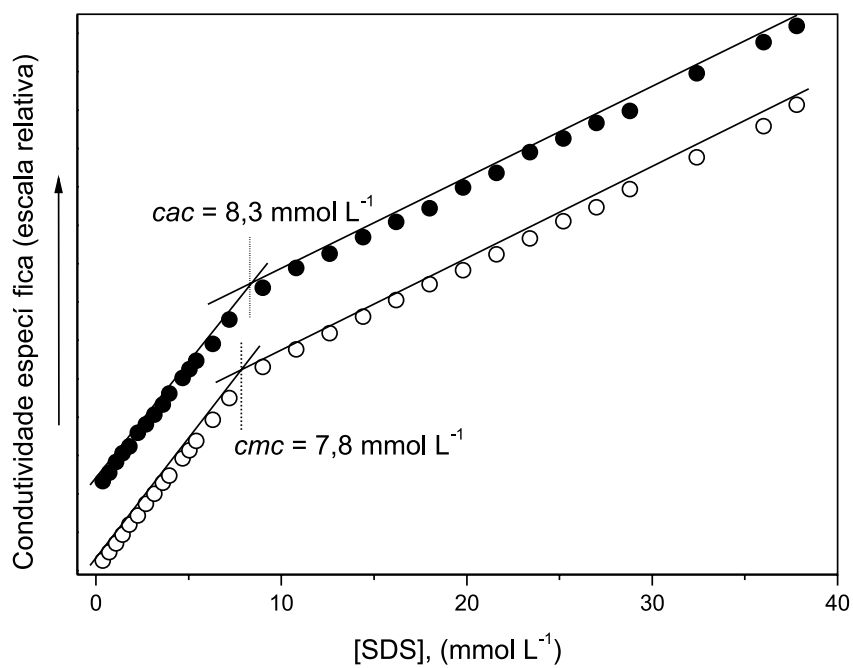

Figura 3. Condutividade específica (em escala relativa) versus concentração de SDS na ausência (O) e na presença $(\bullet)$ de $0,20 \mathrm{mg} \mathrm{mL}^{-1}$ de CNT em solução aquosa

\section{Sistema CNT + Tween 80}

Tensão superficial

O perfil de tensão superficial versus concentração de Tween 80 na presença de $0,20 \mathrm{mg} \mathrm{mL}^{-1}$ de CNT em solução aquosa é apresentado na Figura 4. O valor encontrado para a $\mathrm{cmc}$ do Tween 80 foi de 0,012 $\mathrm{mmol} \mathrm{L}^{-1}$ (Figura $2 \mathrm{~S}$, material suplementar), coerente com a faixa de valor $\left(c m c=0,007-0,012 \mathrm{mmol} \mathrm{L}^{-1}\right)$ descrito na literatura. ${ }^{32} \mathrm{Com}$ o nanotubo de carbono presente na solução, aumenta em mais de 8 vezes o valor da $c a c$ que foi determinado em $0,10 \mathrm{mmol} \mathrm{L}^{-1}$. O efeito da interação do Tween 80 na superfície do nanotubo de carbono é mais intenso do que o visto com o SDS. Por ser um surfactante sem carga, não apresenta a repulsão na parte hidrofílica, como é o caso do SDS. Desta forma, mais moléculas de surfactante interagem na superfície da parede do nanotubo. A eficiência da adsorção na superfície do nanotubo e, consequentemente, o poder de dispersão dos surfactantes são intensamente afetados pelo comprimento da cauda. Caudas maiores significam maior volume espacial e impedimento estérico, promovendo forças repulsivas entre os nanotubos, ${ }^{8}$ fato este evidenciado na dispersão de CNT-Tween 80 obtida neste estudo.

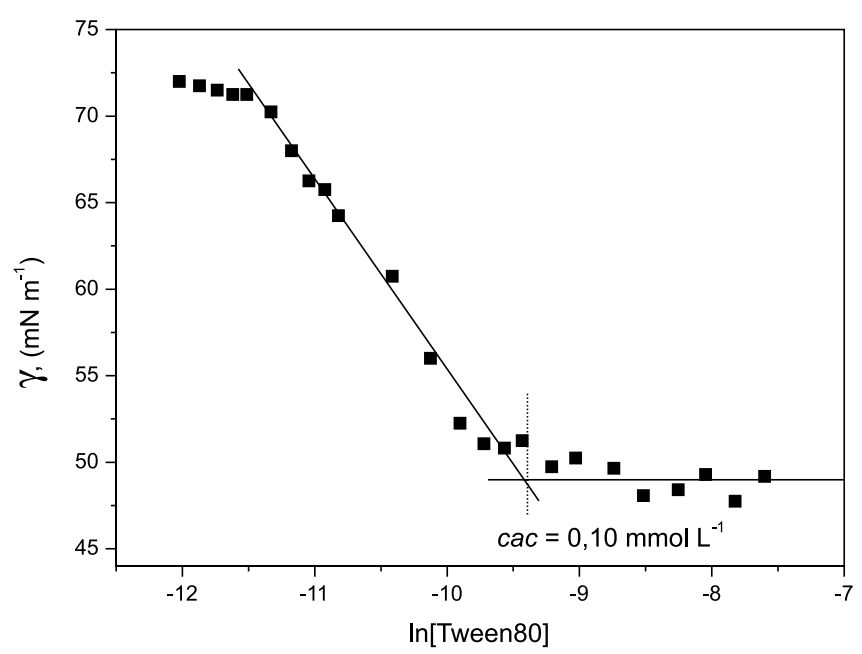

Figura 4. Tensão superficial versus concentração de Tween 80 na presença de 0,20 $\mathrm{mg} \mathrm{mL}^{-1}$ de CNT em solução aquosa

Foram calculados os parâmetros de adsorção interfacial para o Tween 80 puro e na presença de CNT, através das Equações 1 e 2, bem como para o SDS puro e na presença de CNT. Para o Tween 80 puro a concentração em excesso na superfície $\Gamma_{\max }=1,70 \times 10^{-6} \mathrm{~mol}$ $\mathrm{m}^{-2}$, próximo ao encontrado por Pérez-Gramatges et al., ${ }^{33}$ e a $\mathrm{A}_{\min }=$ $97,30 \AA^{2}$ molécula ${ }^{-1} \cdot{ }^{34} \mathrm{~A}$ presença de $0,2 \mathrm{mg} \mathrm{mL}^{-1}$ de $\mathrm{CNT}$ alterou bastante o sistema, obtendo-se o valor de $\Gamma_{\max }=3,64 \times 10^{-6} \mathrm{~mol} \mathrm{~m}^{-2}$, consequentemente a área mínima por molécula diminuiu à metade, $\mathrm{A}_{\text {min }}=45,6 \AA^{2}$ molécula $^{-1}$. Neste sistema a presença de CNT teve maior influência na adsorção interfacial do que no sistema CNT-SDS, a área ocupada por molécula tem uma diminuição de mais de $50 \%$ comparada com o Tween 80 puro, evidenciando um melhor empacotamento de moléculas de Tween 80 na superfície.

\section{Fluorescência}

A técnica de fluorescência utilizando pireno como sonda tem sido amplamente utilizada para monitorar a formação de agregados e determinar a concentração micelar crítica $(\mathrm{cmc})$ de $\operatorname{surfactantes}^{35} \mathrm{e}$ a concentração de agregação crítica ( $c a c$ ) de complexos formados por polímeros-surfactante. ${ }^{29,31,36}$ No entanto, esta técnica pode interferir na obtenção de valores de $\mathrm{cmc}$ e $c a c$, pela possível perturbação da estrutura micelar, decorrente da baixa solubilidade do pireno, formando agregados pré-micelares. ${ }^{29,37}$ A Figura 5 mostra o perfil da razão de $\mathrm{I}_{1} / \mathrm{I}_{3}$ versus a concentração de Tween 80 na ausência e na presença de $0,02 \mathrm{mg} \mathrm{mL}^{-1}$ de CNT. Os valores de $c m c$ foram obtidos no final do primeiro platô do perfil de $\mathrm{I}_{1} / \mathrm{I}_{3}$ versus [Tween 80 ]. ${ }^{29}$ De acordo com esta metodologia, o valor de $c m c$ para o Tween 80 foi de 0,004 
mmol L ${ }^{-1}$, menor do que a encontrada na literatura; ${ }^{32}$ uma explicação é que o pireno possa ter induzido a formação da micela de Tween 80 .

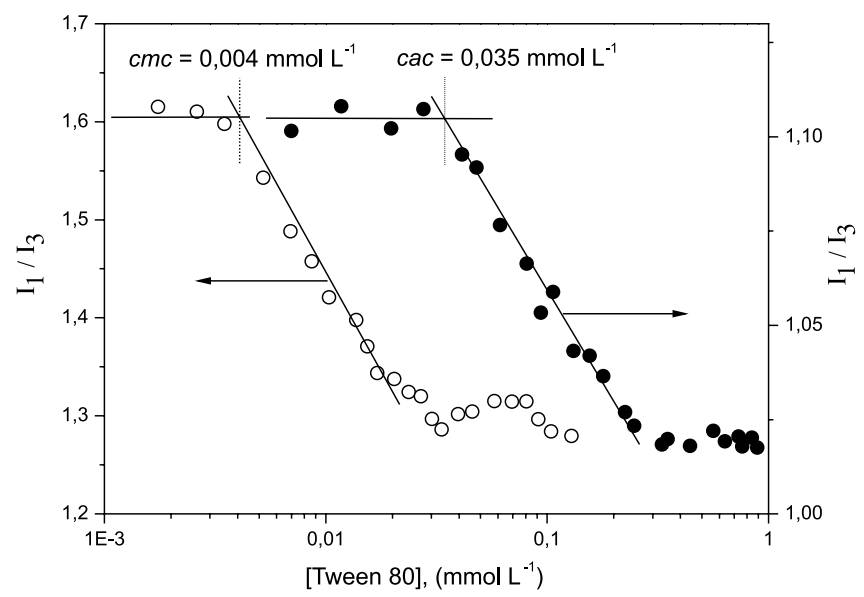

Figura 5. $I_{1} / I_{3}$ versus concentração de Tween 80 na ausência $(O)$ e na presença (•) de 0,20 $\mathrm{mg} \mathrm{mL}^{-1}$ de CNT em solução aquosa contendo 1,0 $\times 10^{-6}$ mol $L^{-1}$ de pireno

A presença de CNT na solução de Tween 80 apresentou um ambiente muito apolar, em baixas concentrações do surfactante a razão $I_{1} / I_{3}$ é de 1,11 . Apesar do meio em que se encontra o pireno ser muito apolar, não afetou o perfil da curva de fluorescência, o valor encontrado da $c a c$ na presença de CNT foi de $0,035 \mathrm{mmol} \mathrm{L}^{-1}$, quase 10 vezes maior do que a $\mathrm{cmc}$ do Tween 80 puro encontrado pelo mesmo método.

\section{Espectroscopia UV-vis}

A fim de se determinar a razão ideal CNT-surfactante para cada surfactante, o experimento foi realizado variando as concentrações de surfactante, enquanto a concentração de nanotubo de carbono foi mantida constante. As dispersões contendo CNT-SDS, CNTTween 80 foram acompanhadas por espectroscopia UV-vis em um comprimento de onda fixo de $260 \mathrm{~nm} .{ }^{12}$ As dispersões de nanotubo de carbono foram feitas utilizando uma concentração fixa de 0,20 $\mathrm{mg} \mathrm{mL}^{-1}$ de CNT, enquanto a concentração de SDS variou de 0,36 a $45,0 \mathrm{mmol} \mathrm{L}^{-1} \mathrm{e}$ a de Tween 80 variou de $1,0 \times 10^{-3}$ a $0,50 \mathrm{mmol} \mathrm{L} \mathrm{L}^{-1}$.

A Figura 6 mostra a absorbância em $260 \mathrm{~nm}$ em função da concentração de SDS. Em baixas concentrações de surfactante, até 4,0 mmol L ${ }^{-1}$, observa-se uma intensidade de absorbância baixa (Abs $0,1)$, devido à pouca dispersão dos CNTs. A partir desta concentração, há um aumento na absorbância, até que a partir de $11,0 \mathrm{mmol} \mathrm{L}^{-1} \mathrm{de}$

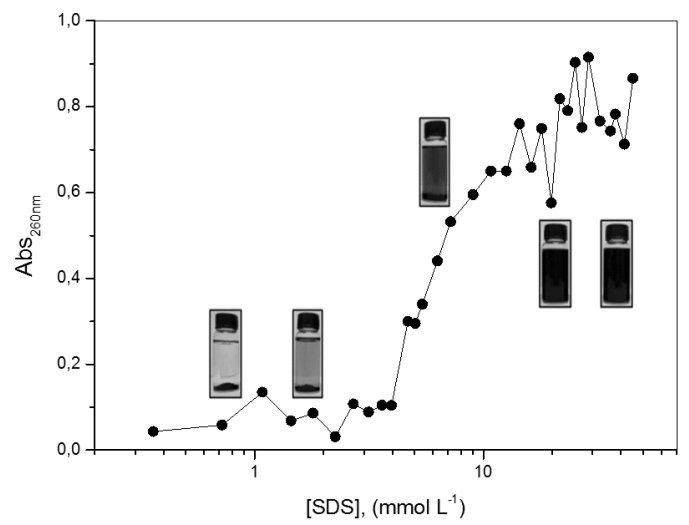

Figura 6. Absorbância em $260 \mathrm{~nm}$ versus concentração de SDS na presença de 0,20 $\mathrm{mg} \mathrm{mL}^{-1}$ de $C N T$
SDS é observada uma boa dispersão dos CNTs na solução, sendo que acima desta concentração a absorbância permanece na faixa de 0,7-0,9. Nas concentrações de SDS em que a absorbância apresenta um patamar, ou seja, apresenta uma boa dispersão do CNT, os valores são próximos dos valores de $c a c$ encontrados pelas técnicas de tensiometria e condutivimetria para o sistema CNT-SDS.

O perfil de absorbância em função da concentração de Tween 80 é apresentado na Figura 7. O aumento da concentração de $1,0 \times 10^{-3}$ até $0,03 \mathrm{mmol} \mathrm{L}^{-1}$ de Tween 80 não apresentou uma boa dispersão dos CNTs; nesta faixa de concentração a absorbância manteve-se constante em $\sim 0,1$, enquanto a concentração $0,04 \mathrm{mmol} \mathrm{L}^{-1}$ de Tween 80 apresentou absorbância de 0,55 . Após atingir esta concentração, a absorbância manteve-se constante na faixa de 0,50 a 0,60 .

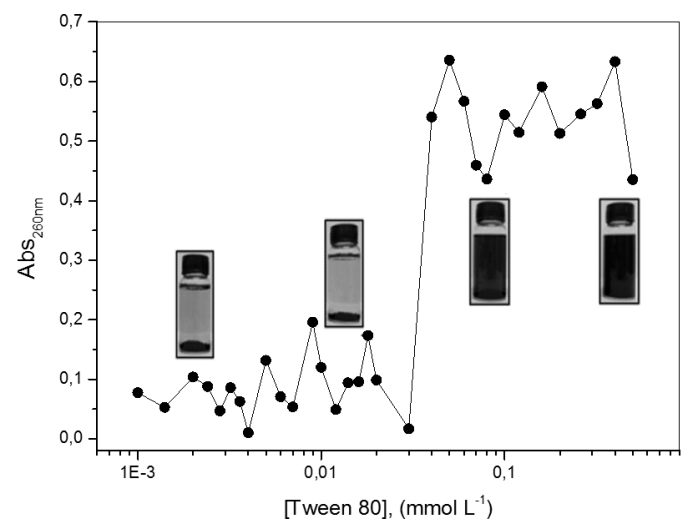

Figura 7. Absorbância em $260 \mathrm{~nm}$ versus concentração de Tween 80 na presença de $0,20 \mathrm{mg} \mathrm{mL}^{-1}$ de CNT

De acordo com as Figuras 6 e 7, a razão ideal CNT-surfactante foi 1:41 para o SDS e 1:3 para o Tween 80 . Rastogi et al. ${ }^{8}$ reportaram uma dispersão estável de CNT (parede múltipla não funcionalizados) com uma razão de 1:520 e 1:400 para SDS e Tween 80, respectivamente. Jiang et al. ${ }^{7}$ encontraram a razão de 1:4 para o SDS, sendo que os CNTs utilizados foram previamente submetidos a tratamento ácido. Yu et al. ${ }^{12}$ alcançaram uma dispersão ideal de 1:1,5 no sistema CNT-SDS, sendo os CNTs de parede múltipla e não funcionalizados. Existe uma discrepância relativamente grande nas concentrações de equilíbrio ideais das dispersões, que pode ser explicada devido à influência da superfície e do comprimento dos nanotubos de carbono, assim como dos métodos e dos equipamentos utilizados para a dispersão. Além disso, a presença de grupos ácidos na superfície, os quais são comumente introduzidos durante a purificação dos nanotubos, poderia aumentar a hidrofilicidade e melhorar a estabilidade da dispersão. ${ }^{10}$ De acordo com as razões obtidas no presente estudo, o Tween 80 foi considerado o melhor surfactante para a dispersão dos nanotubos de carbono quando comparado ao SDS.

\section{CONCLUSÃO}

Foram estudadas dispersões de nanotubos de carbono em soluções aquosas de surfactantes (aniônico e não iônico). Os estudos focaram a interação dos surfactantes com os CNTs, demonstrando que a presença do nanotubo nos sistemas influenciou a concentração micelar crítica dos surfactantes; para ambos a $\mathrm{cmc}$ se deslocou para valores maiores, sendo que para o Tween 80 este aumento foi mais acentuado.

Nós encontramos dispersões de CNTs em soluções aquosas de surfactantes, nas razões de 1:41 e 1:3 para o SDS e Tween 80, respectivamente. Desta forma, conhecendo estas razões pode-se utilizar apenas a concentração de surfactante necessária para recobrir a superfície do nanotubo, evitando o uso de concentrações maiores, que 
poderiam restringir a utilização destas suspensões, além de aumentar o custo do processo.

\section{MATERIAL SUPLEMENTAR}

As Figuras $1 \mathrm{~S}$ e $2 \mathrm{~S}$ mencionadas no texto encontram-se disponíveis gratuitamente, sob a forma de arquivo PDF em http://quimicanova.sbq.org.br.

\section{AGRADECIMENTOS}

Ao $\mathrm{CNPq}$ e à CAPES pelo suporte financeiro, ao Prof. Dr. E. Minatti pela análise de fluorescência.

\section{REFERÊNCIAS}

1. Iijima, S.; Nature 1991, 354, 56.

2. Baughman, R. H.; Zakhidov, A. A.; de Heer, W. A.; Science 2002, 297, 787.

3. Sahoo, N. G.; Rana, S.; Cho, J. W.; Li, L.; Chan, S. H.; Prog. Polym. Sci. 2010, 35, 837 .

4. Chen, W.; Tao, X.; Xue, P.; Cheng, X.; Appl. Surf. Sci. 2005, 252, 1404.

5. Lisunova, M. O.; Lebovka, N. I.; Melezhyk, O. V.; Boiko, Y. P.; J. Colloid Interface Sci. 2006, 299, 740.

6. Ham, H. T.; Choi, Y. S.; Chung, I. J.; J. Colloid Interface Sci. 2005, 286 , 216.

7. Jiang, L.; Gao, L.; Sun, J.; J. Colloid Interface Sci. 2003, $260,89$.

8. Rastogi, R.; Kaushal, R.; Tripathi, S. K.; Sharma, A. L.; Kaur, I.; Bharadwaj, L. M.; J. Colloid Interface Sci. 2008, 328, 421.

9. Madni, I.; Hwang, C.-Y.; Park, S.-D.; Choa, Y.-H.; Kim, H.-T.; Colloids Surf., A 2010, 358, 101.

10. Bystrzejewski, M.; Huczko, A.; Lange, H.; Gemming, T.; Büchner, B.; Rümmeli, M. H.; J. Colloid Interface Sci. 2010, 345, 138.

11. Yurekli, K.; Mitchell, C. A.; Krishnamoorti, R.; J. Am. Chem. Soc. 2004, $126,9902$.

12. Yu, J.; Grossiord, N.; Koning, C. E.; Loos, J.; Carbon 2007, 45, 618.

13. Angelikopoulos, P.; Gromov, A.; Leen, A.; Nerushev, O.; Bock, H.; Campbell, E. E. B.; J. Phys. Chem. C 2010, 114, 2.

14. Rausch, J.; Zhuang, R.-C.; Mäder, E.; Comp. Part A: Appl. Sci. Manufac. 2010, 41, 1038.
15. Whitsitt, E. A.; Barron, A. R.; Nano Lett. 2003, 3, 775.

16. Han, Z.; Zhang, F.; Lin, D.; Xing, B.; Environ. Sci. Technol. 2008, 42, 6869.

17. Bai, Y.; Lin, D.; Wu, F.; Wang, Z.; Xing, B.; Chemosphere 2010, 79, 362.

18. Vaisman, L.; Wagner, H. D.; Marom, G.; Adv. Colloid Interface Sci. 2006, 128,37 .

19. Shaw, D. J.; Introdução à Química de Colóides e de Superfície, Edgard Blücher: São Paulo, 1975.

20. Grossiord, N.; van der Schoot, P.; Meuldijk, J.; Koning, C. E.; Langmuir 2007, 23, 3646.

21. Chen, L.-J.; Lin, S.-Y.; Chern, C.-S.; Wu, S.-C.; Colloids Surf., A 1997, $122,161$.

22. Modolon, S. de M.; Dal Bó, A. G.; Felippe, A. C.; Minatti, E.; Zanette, D.; Quim. Nova 2009, 32, 2046.

23. Rizzatti, I. M.; Zanette, D. R.; Mello, L. C.; Quim. Nova 2009, 32, 518.

24. Lin, S.-Y.; Lin, Y.-Y.; Chen, E.-M.; Hsu, C.-T.; Kwan, C.-C.; Langmuir 1999, 15, 4370.

25. Matarredona, O.; Rhoads, H.; Li, Z.; Harwell, J. H.; Balzano, L.; Resasco, D. E.; J. Phys. Chem. B 2003, 107, 13357.

26. Dong, B.; Su, Y.; Liu, Y.; Yuan, J.; Xu, J.; Zheng, L.; J. Colloid Interface Sci. 2011, 356, 190.

27. Richard, C.; Balavoine, F.; Schultz, P.; Ebbesen, T. W.; Mioskowski, C.; Science 2003, 300, 775.

28. Goddard, E. D.; J. Colloid Interface Sci. 2002, 256, 228.

29. Zanette, D.; Felippe, A. C.; Schweitzer, B.; Dal Bó, A.; Lopes, A.; Colloids Surf., A 2006, 279, 87.

30. Zanette, D.; Frescura, V. L. A.; J. Colloid Interface Sci. 1999, 213, 379.

31. Felippe, A. C.; Bellettini, I. C.; Eising, R.; Minatti, E.; Giacomelli, F. C.; J. Braz. Chem. Soc. 2011, 22, 1539.

32. le Maire, M.; Champeil, P.; Møller, J. V.; Biochim. Biophys. Acta, Biomembr. 2000, 1508, 86.

33. Ruiz-Peña, M.; Oropesa-Nuñez, R.; Pons, T.; Louro, S. R. W.; Pérez-Gramatges, A.; Colloids Surf., B 2010, 75, 282.

34. Canto, G. S. d.; Treter, J.; Yang, S.; Borré, G. L.; Peixoto, M. P. G.; Ortega, G. G.; Braz. J. Pharm. Sci. 2010, 46, 237.

35. Eising, R.; Morés, S.; Bellettini, I. C.; Felippe, A. C.; Dal-Bó, A. G.; Zanette, D.; Quim. Nova 2008, 31, 2065.

36. Felippe, A. C.; Schweitzer, B.; Dal Bó, A. G.; Eising, R.; Minatti, E.; Zanette, D.; Colloids Surf., A 2007, 294, 247.

37. Gouin, S.; Zhu, X. X.; Langmuir 1998, 14, 4025. 


\section{ESTUDO DAS DISPERSÕES AQUOSAS DE NANOTUBOS DE CARBONO UTILIZANDO DIFERENTES} SURFACTANTES

Isabella R. da Silva e Pedro L. M. Barreto*

Departamento de Ciências e Tecnologia de Alimentos, Universidade Federal de Santa Catarina, 88040-900 Florianópolis - SC, Brasil Ismael C. Bellettini

Departamento de Química, Universidade Federal de Santa Catarina, 88040-900 Florianópolis - SC, Brasil

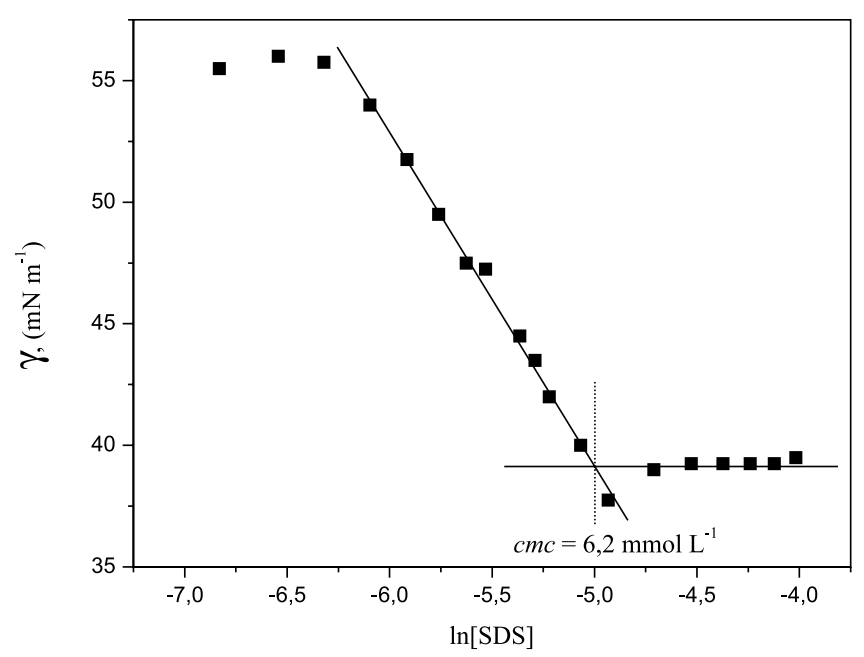

Figura 1S. Tensão superficial versus concentração de SDS em solução aquosa

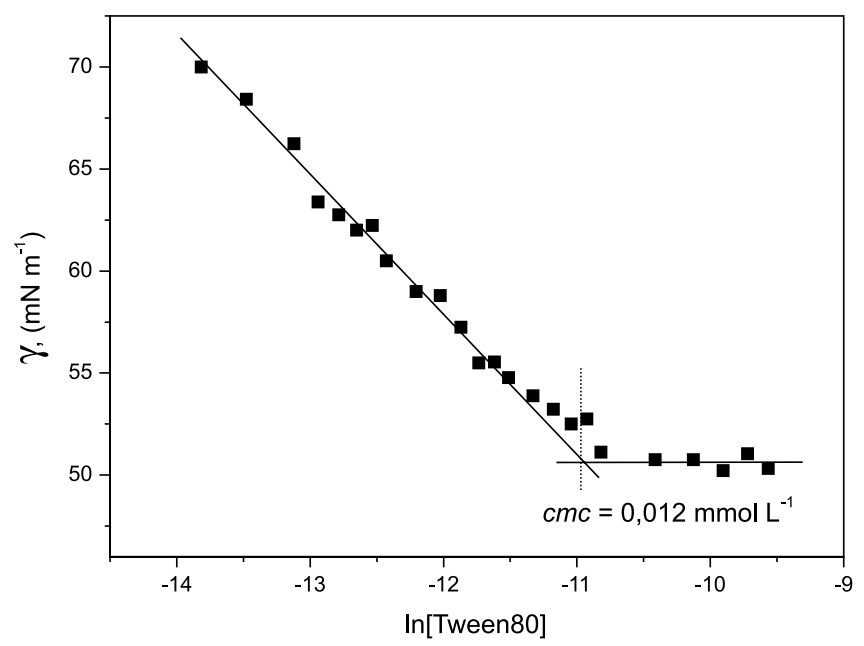

Figura 2S. Tensão superficial versus concentração de Tween 80 em solução aquosa

*e-mail: barreto@cca.ufsc.br 